\title{
The use of the EVITA algorithm for clinical assessment of novel agents used in prostate cancer, metastatic melanoma, and systemic lupus erythematosus
}

\author{
Thomas D. Szucs • Daniele Puri • Patricia R. Blank
}

Received: 5 February 2014 / Accepted: 13 May 2014 / Published online: 27 May 2014

(C) Springer-Verlag Berlin Heidelberg 2014

\begin{abstract}
Purpose Existing health technology assessment methods can be time-consuming and complicated to use in practice. EValuation of pharmaceutical Innovations with regard to Therapeutic Advantage (EVITA) is a recently developed drug assessment strategy that provides a detailed and clinically relevant evaluation of new agents compared to standard therapies. We therefore sought to use EVITA to evaluate eight novel agents recently introduced to clinical practice or in late-stage trials for the treatment of prostate cancer, metastatic melanoma, or systemic lupus erythematosus (SLE).

Methods Eight agents (abiraterone, enzalutamide, sipuleucelT, Prostvac, radium 223, ipilimumab, vemurafenib, and belimumab) were selected for study using the EVITA algorithm. A comprehensive literature search was performed to find clinical trial data, which were then classified using the EVITA protocol. EVITA was also compared to results from health technology assessments (HTAs) or reimbursement decisions. Results The EVITA scores for the eight drugs ranged from 5.5 to 9: all the selected agents are therefore classed as 'recommended' and are likely to produce a therapeutic advantage. In particular, vemurafenib is likely to be highly beneficial to patients with metastatic melanoma and radium 223 to patients with metastatic prostate cancer affecting the bone. The EVITA results were generally concordant with HTAs.
\end{abstract}

Electronic supplementary material The online version of this article (doi:10.1007/s00228-014-1698-6) contains supplementary material, which is available to authorized users.

T. D. Szucs $(\bowtie) \cdot$ D. Puri $•$ P. R. Blank

Institute of Pharmaceutical Medicine (ECPM), University of Basel,

Klingelbergstrasse 61, 4056 Basel, Switzerland

e-mail: thomas.szucs@unibas.ch

\section{P. R. Blank}

Institute of Social- and Preventive Medicine, Medical Economics, University of Zurich, Hirschengraben 84, 8001 Zurich, Switzerland
Conclusions All the agents show favourable EVITA scores and are therefore recommended for clinical practice. EVITA is an easy-to-use tool that provides clinical context to the assessment of newly introduced agents and can be easily used by non-specialists.

Keywords EVITA · Health technology assessment . Metastatic melanoma $\cdot$ Prostate cancer $\cdot$ Systemic lupus erythematosus

\section{Introduction}

Many new therapeutic agents introduced to the market claim to be innovative, but their actual clinical benefit is often uncertain at the time of regulatory approval [1]. Several multifactorial health technology assessment methods are used to evaluate the likely benefit of new therapies, such as those issued by the National Institute for Health and Clinical Excellence (NICE) in the UK, the German Institut für Qualität und Wirtschaftlichkeit im Gesundheitswesen, and the evaluation strategy proposed by Caprino and Russo [2]. These assessments are comprehensive but time-consuming and can be complicated for non-specialists to use. Other evaluation strategies, such as the classification by Fricke and Klaus [3], do not require new agents to show a clinically significant advantage over standard treatments in order to be deemed 'innovative'. There is a need for a simple and transparent tool that evaluates the benefits and risks of new drugs over existing treatments.

EValuation of pharmaceutical Innovations with regard to Therapeutic Advantage (EVITA) is a recently developed drug assessment strategy that provides a time-oriented and detailed evaluation of new agents compared to standard therapies [1]. Data from eligible randomised controlled trials are used in the EVITA algorithm, which takes into account an efficiency profile, a risk profile, and a trial setting, the latter being used 
to contextualise the clinical validity of the efficiency and risk profile results. The overall numerical result is visualised as a colour-coded bar graph that shows the benefit of the new treatment and is easily interpretable, even by non-specialists. Another advantage of EVITA is that since the algorithm does not depend on the chemical composition and definition of a new chemical entity (NCE), it can be applied to any new therapeutic modality introduced into clinical practice, including small molecule inhibitors, immunomodulatory agents, or radionuclides.

Although EVITA has been used to assess a few commonly used drugs ([1] and http://www.hta.uni-bremen.de/index.php/ projekte/evita/evita-english), the algorithm has yet to be applied to cutting-edge agents that are emerging as potential therapies for life-threatening diseases.

Here, we use EVITA to evaluate eight recently introduced agents (abiraterone, enzalutamide, sipuleucel-T, Prostvac, radium 223, ipilimumab, vemurafenib, and belimumab) already approved for clinical use or still in phase III trials; these agents are used to treat castration-resistant prostate cancer, metastatic melanoma, or systemic lupus erythematosus (SLE). The primary objective of this study is to assess the benefits and risks of new drugs compared to existing standard treatments and the secondary objective is to determine the practicality and validity of EVITA by comparing our results to existing Health Technology Assessment (HTA) reports.

\section{Materials and methods}

Eight agents (abiraterone, enzalutamide, sipuleucel-T, Prostvac, radium 223, ipilimumab, vemurafenib, belimumab) were selected for study using EVITA. Approval of the local ethics committee was not required since the study did not directly require the participation of human study subjects (EKBB).

EVITA was conducted as described in [1]. EVITA requires input of clinical trial data with a Jadad score (used to assess the methodological quality of a clinical trial [4]) of at least three. A systematic literature search of the eight study drugs was first conducted using PubMed and Google Scholar. The EVITA algorithm comprises four main steps for each drug: (1) classification of the main therapeutic aim of the new agent (prevention or treatment), with treatment then being divided into four categories (Table 1); (2) calculation of the absolute risk reduction (ARR; in this study, the ARR in OS after 1 year) or the numbers needed to treat (NNT) for each therapy (i.e. the new agent versus standard treatment or placebo) to produce a modifier score (Table 2); (3) comparison of the efficiency of the two treatments (new agent versus standard treatment or placebo; Table 3), with the efficiency score then being calculated by adding the modifier to the outcome results (agents showing benefit in multiple trials have higher scores); and
Table 1 Therapeutic aim and disease category

\begin{tabular}{ll}
\hline Prevention & To reduce risk of disabling or impairing events \\
\hline Treatment & $\begin{array}{l}\text { To cure diseases, to substitute missing substances } \\
\text { indispensable to life, to modify or relieve symptoms } \\
\text { Severity grading of the diseases: }\end{array}$ \\
& $\begin{array}{l}\text { I. acute life-threatening or severe chronic disease } \\
\text { II. rehabilitation }\end{array}$ \\
& III. less severe acute or chronic disease \\
IV. application outside a treatment context
\end{tabular}

finally (4) comparing the adverse effects exhibited by the two therapies (new agent versus standard treatment or placebo) with each grade of adverse effect (1-5) to derive a risk score for both groups (Table 4). The overall score is the sum of the efficiency score and the risk score, which is then visualised as a colour-coded bar graph (see examples in Fig. 2). Since the design of each study varied, each study needed to be assigned a trial setting using the flowchart shown in Fig. 1. Additionally, each drug was described in an EVITA datasheet for easy reference (Supplementary File 1).

The EVITA scores of the eight drugs studied were compared to the Health Technology Assessment (HTA) reports and reimbursement decision reports available on the Turning Research Into Practice (TRIP) database (http:// www.tripdatabase.com/). Each concordant or discordant result was discussed.

\section{Results}

\section{Overall findings}

Each drug was evaluated separately and a summary EVITA assessment compiled for each agent (Fig. 2 and Supplementary File 1). The EVITA scores for the eight drugs ranged from

\begin{tabular}{llll}
\cline { 2 - 3 } Table 2 Modifier & NNT & ARR & Modifier \\
\cline { 2 - 4 } & Prevention & & \\
& $<20$ & $5-100 \%$ & 2.0 \\
& $20-<50$ & $<5 \%$ & 1.75 \\
& $50-<100$ & $<2 \%$ & 1.5 \\
& $100-<175$ & $<1 \%$ & 1.25 \\
$175-<300$ & $<0.57 \%$ & 1.0 \\
& $300-<500$ & $<0.33 \%$ & 0.75 \\
& $500-<1,000$ & $<0.2 \%$ & 0.5 \\
& $\geq 1,000$ & $<0.1 \%$ & 0.25 \\
& Treatment & & \\
& $<3$ & $>30 \%$ & 2.0 \\
ARR absolut risk & $3-<10$ & $10-30 \%$ & 1.5 \\
reduction, $N N T$ number & $\geq 10$ & $<10 \%$ & 1.0 \\
needed to treat &
\end{tabular}


Table. 3 Efficiency profile

\begin{tabular}{llll}
\hline RCTs showing evidence of & $\begin{array}{l}\text { n. of } \\
\text { RCT }\end{array}$ & $\begin{array}{l}\text { p. rel. } \\
\text { outcome }\end{array}$ & $\begin{array}{l}\text { Surr. } \\
\text { outcome }\end{array}$ \\
\hline Superiority & 0 & 0 & 0 \\
& 1 & +5 & +2.5 \\
& $\geq 2$ & +7.5 & +3.75 \\
Non-inferiority/equivalence & 0 & 0 & 0 \\
$\quad$ (in the presence of other & 1 & -1.67 & -0.83 \\
RCT showing superiority) & $\geq 2$ & -2.5 & -1.25 \\
Non-inferiority/equivalence & any & 0 & 0 \\
$\quad$ (in the absence of other & & & \\
RCT) & 0 & 0 & 0 \\
Non-inferiority/equivalence & 1 & +1.67 & +0.83 \\
$\quad$ in the presence of other & $\geq 2$ & +2.5 & +1.25 \\
RCT showing inferiority) & 0 & 0 & 0 \\
Inferiority & 1 & -5 & -2.5 \\
& $\geq 2$ & -7.5 & -3.75 \\
Sum & & & \\
Modifier & & & \\
Efficiency score & & & \\
\hline
\end{tabular}

5.5 to 9 (Fig. 2), i.e. all the selected agents are recommended. The 'therapeutic aim' (step 1) for all the drugs under study was treatment category 1 , since all had been developed for life-threatening chronic diseases. The efficiency scores ranged from 6 to 9, which was in part due to the different numbers of randomised studies available and slightly different modifiers (ranging from 1 to 1.5 ). Each study had a significant patientrelevant outcome. All risk scores were 0 with the exception of vemurafenib and ipilimumab, which had risk scores of 0.5 and -1 , respectively, since they did not have a frequency above $10 \%$ for every grade of adverse event. There was one A 1 trial setting (vemurafenib), five A2 settings (abiraterone, ipilimumab, enzalutamide, radium 223, and belimumab), and two question marks (Prostvac and sipuleucel-T), since these two therapies were not compared to the standard treatment or with placebo as an add-on to the standard therapy; these results are therefore of questionable validity.

The use of EVITA was generally time efficient and suitable for non-specialists, with the most time-consuming element being literature searching and interpretation. Overall, 25 different studies and reviews were analysed in this study. Calculation of the modifier was not always trivial since the ARRs and NNT were not always directly available; in these studies, the data of the patients in both treatment arms (new agent versus standard treatment or placebo) still alive at 1 year were extracted and the 1-year ARR and OS calculated. Accurately identifying the separate frequencies of the grades 3-5 adverse events was sometimes difficult since they were often described together; in these cases, the frequencies of the adverse effects of the new drugs were nearly the same as the ones of the standard treatments or placebo, and therefore their distribution over grades 3-5 were assumed to be the same (risk score 0).

With respect to validity, the EVITA results were compared with HTA outcomes or drug reimbursement decisions from the TRIP database (Supplementary File 2). Most of the reports were positive and concordant with EVITA. Negative results were mainly based on inappropriate or missing dossiers or an unfavourable health economic analysis. However, EVITA does not include economic data in its assessment and can therefore only determine the clinical benefit of a new drug. In cases where no decision had yet been made by the decisionmaking bodies (Sipuleucel-T and Prostvac), EVITA already had a question mark (i.e. questionable validity) for the trial setting.

Specific agents

\section{Abiraterone}

One randomised double-blind phase III study of abiraterone was identified [5]. In this study, abiraterone

Table 4 Risk profile

\begin{tabular}{|c|c|c|c|}
\hline Severity grading & Frequency & Ther. inv. & Ther. stand. \\
\hline \multicolumn{4}{|l|}{ Adverse events (AE) } \\
\hline \multirow{5}{*}{$\begin{array}{l}\text { Grades } 5+4 \\
\text { (death related to } \mathrm{AE} \text { or } \\
\text { life-threatening } \mathrm{AE} \text { or } \\
\text { disabling } \mathrm{AE} \text { ) }\end{array}$} & $\geq 10 \%$ & -4 & -4 \\
\hline & $\geq 1 \%$ & -3 & -3 \\
\hline & $\geq 0.1 \%$ & -2 & -2 \\
\hline & $<0.1 \%$ & -1 & -1 \\
\hline & 0 & 0 & 0 \\
\hline \multicolumn{4}{|l|}{ Grade 3} \\
\hline \multirow[t]{5}{*}{ (severe and undesirable AE) } & $\geq 10 \%$ & -2.5 & -2.5 \\
\hline & $\geq 1 \%$ & -2 & -2 \\
\hline & $\geq 0.1 \%$ & -1 & -1 \\
\hline & $<0.1 \%$ & 0 & 0 \\
\hline & 0 & 0 & 0 \\
\hline \multicolumn{4}{|l|}{ Grades $2+1$} \\
\hline \multirow[t]{5}{*}{ (moderate $\mathrm{AE}$ or mild $\mathrm{AE}$ ) } & $\geq 10 \%$ & -1.5 & -1.5 \\
\hline & $\geq 1 \%$ & -1 & -1 \\
\hline & $\geq 0.1 \%$ & -0.5 & -0.5 \\
\hline & $<0.1 \%$ & 0 & 0 \\
\hline & 0 & 0 & 0 \\
\hline \multicolumn{4}{|l|}{ Interactions } \\
\hline \multicolumn{2}{|c|}{ Frequent or serious clinical consequence } & -2 & -2 \\
\hline \multicolumn{2}{|c|}{ Occasional or may have clin. consequence } & -1.5 & -1.5 \\
\hline \multicolumn{2}{|c|}{ Dose change } & -1 & -1 \\
\hline \multicolumn{2}{|c|}{ Unlikely/probably or no clin. consequence } & 0 & 0 \\
\hline \multicolumn{2}{|c|}{ No information available } & -1 & -1 \\
\hline \multicolumn{4}{|l|}{ Sum } \\
\hline \multicolumn{4}{|l|}{ Risk score } \\
\hline
\end{tabular}

ther. inv. therapy investigated, ther. stand. therapeutic standard 


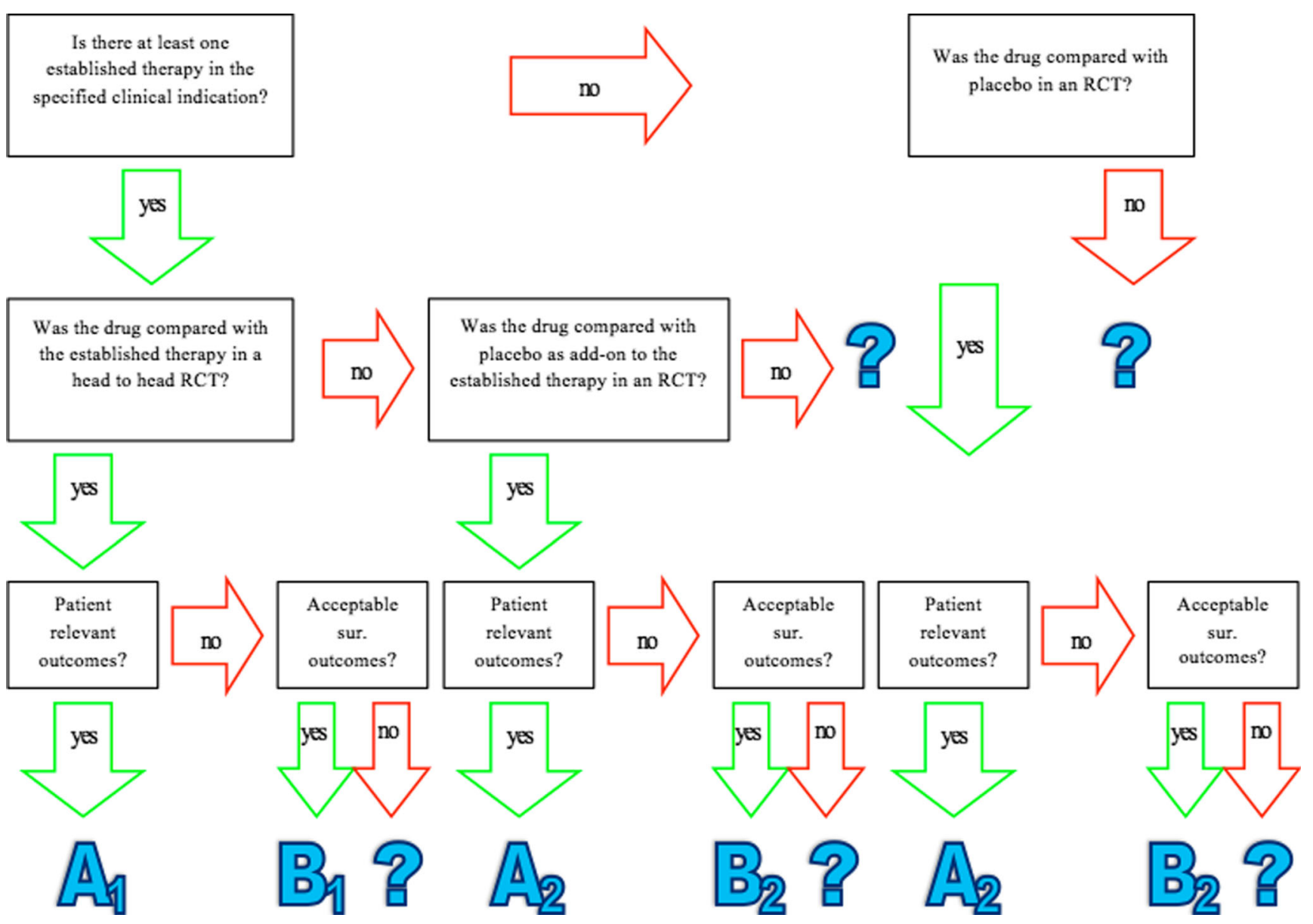

Fig. 1 Flowchart showing the decision tree used to define EVITA trial settings

was compared to placebo as an add-on to the standard treatment (docetaxel); the trial setting was therefore A2. Abiraterone is indicated for prolonging the lifespan of patients with castration-resistant prostate cancer; the treatment category was therefore 1 . The ARR in OS at 1 year was $13 \%$, resulting in a modifier of 1.5 . The primary endpoint was median OS, which was significantly longer in the abiraterone cohort than in the placebo cohort (15.8 versus 11.2 months), earning an efficiency score of 6.5 . The adverse effects of both treatments were similar, thus the risk score was 0 , resulting in a final score of 6.5 .

\section{Radium 223}

A randomised, multicentre, placebo-controlled phase II study [6] and a phase III randomised trial [7] were identified; in both of these, radium 223 was compared with placebo. Since there was no standard treatment for the specific indication (treatment of bone metastases in castration-resistant prostate cancer), the trial setting was A2. Since radium 223 should improve both the quality and quantity of life, the treatment category was 1 . The ARR in OS at 50 weeks (no data were available at 1 year) was $16 \%$, resulting in a modifier of 1.5 . Both studies revealed a significantly improved median OS (phase II: 15 versus 10.7 months; phase III 14 versus 11.2 months), earning an efficiency score of 9 . The adverse effects of both treatments were similar thus the risk score was 0 , resulting in a final score of 9 .

\section{Enzalutamide}

One randomised, double-blind phase III study was found [8] in which enzalutamide was compared to placebo as add-on to the standard treatment (docetaxel), a trial setting of A2. Since enzalutamide was developed to prolong the lifespan of patients with castration-resistant prostate cancer, the treatment category was 1 . The ARR in OS at 1 year was $30 \%$, resulting in a modifier of 1.5. The primary endpoint was defined as the median OS, which was significantly better in the enzalutamide group than in the placebo group (18.4 versus 13.6 months), earning an efficiency score of 6.5 . The adverse effects of both treatments were similar, thus the risk score was 0 , resulting in a final score of 6.5 . 


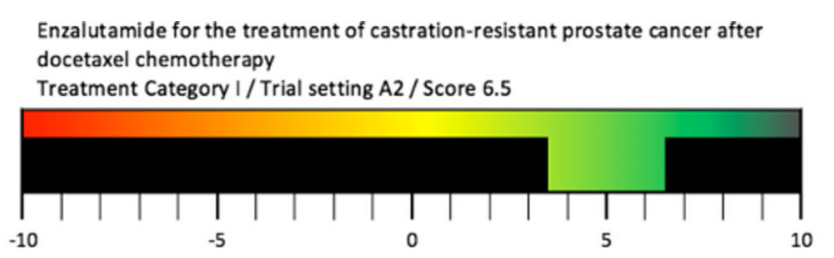

Abiraterone for the treatment of castration-resistant prostate cancer after docetaxel chemotherapy

Treatment Category 1 / Trial setting A2 / Score 6.5

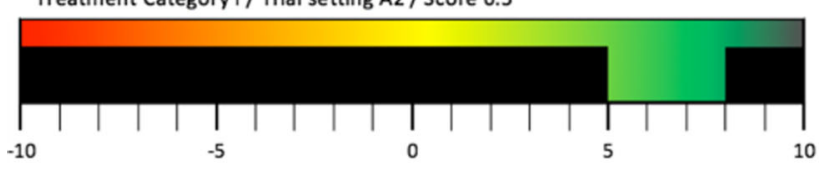

Radium 223 for the treatment of bone metastases in castration-resistant prostate cancer

Treatment Category I / Trial setting A2 / Score 9

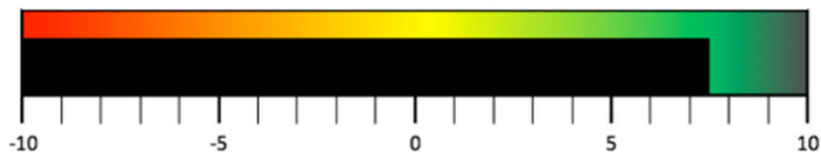

Prostvac-VF for the treatment of castration-resistant prostate cancer

Treatment Category I / Trial setting "?" / Score 6

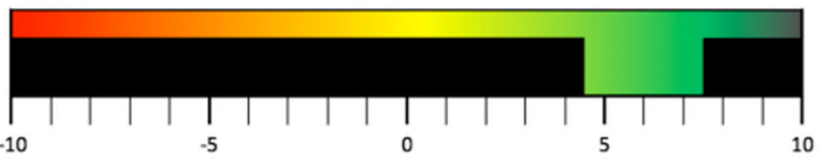

Sipuleucel-T for the treatment of castration-resistant prostate cancer

Treatment Category I/ Trial setting "?" / Score 8.5

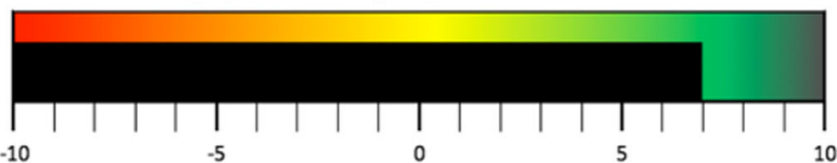

Ipilimumab for the treatment of previously untreated metastatic melanoma Treatment Category I / Trial setting A2 / Score 5.5

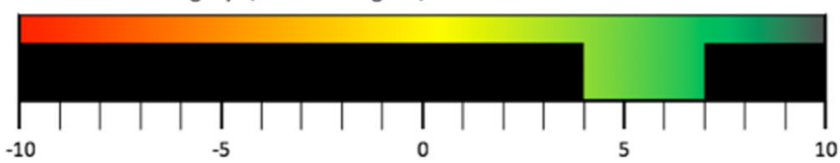

Vemurafenib for the treatment of previously untreated stage IIIC or stage IV melanoma that tested positive for the BRAFV600E mutation

Treatment Category I / Trial setting A1 / Score 7

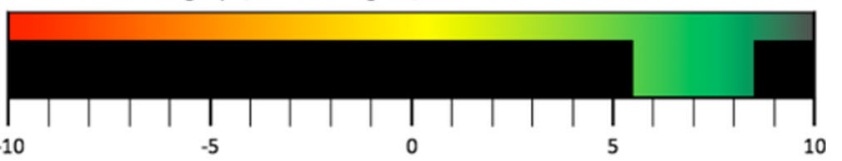

Belimumab for patients with active systemic lupus erythematosus (SLE) Treatment Category I / Trial setting A2 / Score 9

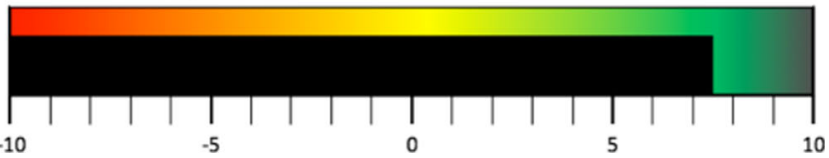

Fig. 2 Summary diagram showing the EVITA scores of the eight agents studied

\section{Sipuleucel-T}

Three randomised, double-blind phase III studies were identified. Two were analysed together, since their study design was identical [9]. Sipuleucel-T was not compared to the standard treatment (docetaxel) or placebo, and therefore, the trial setting was a question mark. The treatment category was defined as 1, since sipuleucel-T should improve survival of patients with castration-resistant prostate cancer. The ARR in OS at 1 year was $7 \%$ in the integrated study [9] and $8 \%$ in the Kantoff et al. [10] study, resulting in a modifier of one. Both studies showed a significant reduction in the RR of death ( $33 \%$ in the integrated study, $22 \%$ in the Kantoff et al. study), earning an efficiency score of 8.5. The adverse effects of both treatments were similar, thus the risk score was 0 , and the final score was 8.5 .

\section{Prostvac}

Two randomised, double-blind phase II studies were identified $[11,12]$. Since the control group was missing in Gulley et al. [12] and all cohorts received Prostvac (with four different doses of immune adjuvants), this study was not included in the present analysis. In the other study [11], Prostvac was not compared to the standard treatment (docetaxel) or to placebo, and therefore, the trial setting was a question mark. Prostvac was developed to prolong the survival of patients with castration-resistant prostate cancer, and therefore, the treatment category was 1 . The ARR in OS at 1 year was $4 \%$, resulting in a modifier of 1 . At three years post-treatment, Prostvac patients had a significantly improved OS (30 versus $17 \%$ ), earning an efficiency score of 6 . The adverse effects of both treatments were similar, thus the risk score was 0 , and the final score was 6.

\section{Ipilimumab}

One randomised, double-blind phase III study was identified [13]. Ipilimumab was compared to placebo as add-on to the standard treatment (dacarbazine), and therefore, the trial setting was A2. The treatment category was classified as 1 , since metastatic melanoma is a life-threatening disease. The ARR in OS at 1 year was $11 \%$, resulting in a modifier of 1.5 . The primary endpoint was defined as the median OS, which was significantly longer in the ipilimumab cohort than in the placebo cohort (11.2 versus 9.1 months), earning an efficiency score of 6.5. The adverse effects of both treatments were similar except for grades 4 and 5 (ipilimumab group $16.2 \%$ and placebo group $9.2 \%$ ); therefore, the risk score was -1 , and the final score was 5.5 . 


\section{Vemurafenib}

One randomised, multinational phase III study was identified [14]. Since vemurafenib was compared to the standard treatment (dacarbazine), the trial setting was A1. The treatment category was classified as 1 , since metastatic melanoma is a life-threatening disease. The ARR in OS at six months was $20 \%$, resulting in a modifier of 1.5 . The OS at 1 year could not be evaluated because the dacarbazine group was allowed to cross over to vemurafenib, since the primary endpoints (OS and progression-free survival (PFS)) had already met the prespecified criteria for statistical significance; the efficiency score was therefore 6.5. The vemurafenib group had a grades 2-3 adverse effects frequency of greater than $10 \%$ and adverse events of grade $4-5$ in less than $1 \%$ of the patients. In the dacarbazine group, there were less grade $3(\geq 1 \%)$ events but more grade 4 and $5(\geq 1 \%)$ events. Therefore, the risk score was 0.5 resulting in a final score of 7 .

\section{Belimumab}

Two randomised, double-blind, phase III studies were identified $[15,16]$; in both studies (at two different doses 10 and $1 \mathrm{mg} / \mathrm{kg}$ ), belimumab was compared to placebo as an add-on to the standard treatment (individually adapted), thus the trial setting was A2. The treatment category was 1 , since SLE is a severe chronic disease. Since there were no OS data, we took the ARR of the SRI (systemic lupus erythematosus responder index), which is based on the overall severity or development of substantial disease activity in new organ systems [17]. The ARRs of the SRI at 1 year were 14 and $12 \%(10 \mathrm{mg} / \mathrm{kg})$ and 9 and $7 \%(1 \mathrm{mg} / \mathrm{kg})$ in the two trials, thus the modifier was 1.5 . Since both studies showed that, significantly, more people were responsive to the $10 \mathrm{mg} / \mathrm{kg}$ belimumab group than in the placebo group; the efficiency score was 9. The adverse effects of both treatments were similar, thus the risk score was 0 , and the final score was 9 .

\section{Discussion}

EVITA is a recently described algorithm designed to assess the efficiency of new agents recently introduced into clinical practice in trials. The methodology is time efficient and simple, yet still detailed enough to produce meaningful and useful results. Here we examine the use of EVITA for eight novel therapies in order to assess their clinical utility using this algorithm.

These particular agents were developed to treat three different diseases: castration-resistant prostate cancer, metastatic melanoma, and systemic lupus erythematosus (SLE); all three of these diseases are acutely life-threatening or severely chronic. Prostate cancer is the third leading cause of male cancer-related death in Europe [18]. Although many men initially respond to androgen-deprivation therapy, progression eventually occurs. Following cytotoxic chemotherapy with docetaxel, the median overall survival (OS) is less than two years [19, 20]; new treatment options are therefore needed. Metastatic melanoma is responsible for three-quarters of all deaths caused by skin cancer [21]. Currently, dacarbazine, the only chemotherapeutic agent approved by the FDA for the treatment of metastatic melanoma, is considered standard treatment, but it is only associated with a median OS of 5.6 to 7.8 months in phase 3 studies [22-25] and has never actually been shown to improve survival in randomised controlled trials [26, 27]. However, ipilimumab and vemurafenib are two new emerging targeted therapies showing clinical promise for metastatic melanoma. Finally, SLE is a chronic autoimmune disorder that markedly impairs patients' quality of life [28]. Although therapies commonly used in clinical practice, such as corticosteroids, hydroxychloroquine, and immunosuppressive drugs, all improve outcomes for patients with SLE, they are associated with significant side effects and more effective treatments would be desirable [15]. Circulating $B$ cell activating factor (BAFF) is commonly elevated in SLE and correlates with disease activity and anti-double-stranded DNA (anti-dsDNA) antibody concentrations [29-31]. Inhibiting BAFF is therefore a novel approach for treating SLE [32].

In contrast to other drug assessment systems, in which new agents do not need to show a clinically significant advantage over standard treatments [1], EVITA compares the new agent with the standard treatment or with placebo, thereby providing clinical relevance and context rather than simply the details of the chemical innovation; proving clinical benefit is ultimately what matters in terms of clinical practice. Important clinical parameters such as adverse effects are included and vary the final result, which is of real clinical importance when assessing therapeutic index in vulnerable and often very ill patients. Assessment of the trial setting is an important parameter to include to assess both the validity of the result and indicate how advanced the drug is in clinical development, providing an indicator of whether more research may be needed for licensing. Additionally, the EVITA protocol is transparent, easily reproducible by anyone with access to the clinical studies, and the final score can easily be adapted when new studies are published. EVITA is therefore a dynamic tool that can be used to chart the progress of drug development during the clinical phases of testing.

In our study, no EVITA result was negative. This might be due to the existence of publication bias, which is known to lead to more positive pivotal trials being published. Puntmann et al. do, however, report negative EVITA results for pioglizone (for diabetes mellitus) and bupropion (for major 
depression) due to a lack of proven superiority of patientrelevant outcomes or an unfavourable risk profile [1].

We did experience some difficulties when using EVITA and it does have some limitations. In cases where there are two studies with the same design conducted in two different groups of patients [e.g. lenalidomide in 1], the original algorithm dictates that these should count as one study, whereas we suggest that in this situation the reproducibility of the result increases the level of confidence in the agent and should therefore carry more weight than a single study. Generally, it is not possible to weight the methodical differences between studies, thus they can be of different quality but still generate the same EVITA score. From a practical perspective, the extraction of grades 4 and 5 adverse events was often difficult since many studies count them with grade 3 adverse events. Generating the ARRs or NNTs was difficult when these measurements were not specifically detailed in the studies and required a manual calculation that might be prone to error.

With respect to the different diseases studied, all five of the newly developed agents for castration-resistant prostate cancer (abiraterone, radium 223, enzalutamide, Prostvac, and sipuleucel-T) had high EVITA scores, indicating that these are highly effective agents. The five agents represent three different treatment modalities: two small molecular inhibitors targeting the androgen pathway (abiraterone and enzalutamide), two tumour vaccines (Prostvac and sipuleucel-T), and one radionuclide (radium 223). This diversity of approach might be important for targeting the disease using multi-modal therapy, and it is important to note that EVITA is capable of producing interpretable and comparable results for very different treatment modalities. Abiraterone, enzalutamide, and radium 223 all appear to be promising agents, since their EVITA scores were 6.5 or above. However, the Prostvac and sipuleucel-T results need interpreting with caution due to the suboptimal nature of the underlying trials (question marks), and further clinical research on these agents is required. This is also in line with the evaluations available in the TRIP database, where many evaluations have yet to decide whether the drug should be recommended or not. However, if the economic analysis was a major reason for a negative response, this will not be accounted for by EVITA and will have no influence on the EVITA result. The EVITA evaluations for these drugs would need to be updated when further data become available.

Both the newly developed agents for metastatic melanoma (ipilimumab and vemurafenib) were effective and could be recommended (ipilimumab 5.5 and vemurafenib 7). Even after 6 months, the vemurafenib-treated group had an ARR in OS of $20 \%$ compared to the placebo group, whereas ipilimumab showed a reduction of $11 \%$ at 1 year. However, these two drugs cannot be directly compared since vemurafenib is given specifically to patients whose tumours harbour a $B R A F$ mutation, whereas ipilimumab is not subdivided on the basis of a molecular test. Therefore, vemurafenib can only be considered of benefit in BRAF mutation carriers while ipilimumab is applicable to the wider population.

Belimumab was highly effective for the treatment of SLE and is highly recommended (EVITA score 9). Other available therapies improve outcomes for patients with SLE but have unfavourable side-effect profiles [15]. Belimumab showed a significant ARR in the SLE Responder Index (SRI) (which takes quality of life into account), and therefore, the patients benefit not only from a prolonged lifespan but also from a higher health-related quality of life. Inhibition of BAFF with belimumab might represent a step forward in the management of patients with SLE.

In conclusion, here, we have applied the newly developed EVITA algorithm to the assessment of eight highly promising new agents being used to treat life-threatening serious or chronic diseases. All the agents show favourable EVITA scores and are therefore recommended for clinical practice. EVITA is an easy-to-use tool that provides clinical context to the assessment of newly introduced agents and can be easily used by non-specialists.

Conflict of interest statement All authors confirm that there are no conflicts of interests.

\section{References}

1. Puntmann I, Schmacke N, Melander A, Lindberg G, Muhlbauer B (2010) EVITA: a tool for the early evaluation of pharmaceutical innovations with regard to therapeutic advantage. BMC Clin Pharmacol 10:5

2. Caprino L, Russo P (2006) Developing a paradigm of drug innovation: an evaluation algorithm. Drug Discov Today 11(21-22):999-1006

3. Fricke U, Klaus W (2008) Neue Arzneimittel - Fakten und Bewertungen von 2004 bis 2006 zugelassenen Arzneimitteln. Wissenschaftliche Verlagsgesellschaft $\mathrm{GmbH}$, Stuttgart

4. Jadad AR, Moore RA, Carroll D, Jenkinson C, Reynolds DJ, Gavaghan DJ, McQuay HJ (1996) Assessing the quality of reports of randomized clinical trials: is blinding necessary? Control Clin Trials 17(1):1-12

5. Fizazi K, Scher HI, Molina A, Logothetis CJ, Chi KN, Jones RJ, Staffurth JN, North S, Vogelzang NJ, Saad F, Mainwaring P, Harland S, Goodman OB Jr, Sternberg CN, Li JH, Kheoh T, Haqq CM, de Bono JS (2012) Abiraterone acetate for treatment of metastatic castration-resistant prostate cancer: final overall survival analysis of the COU-AA-301 randomised, double-blind, placebo-controlled phase 3 study. Lancet Oncol 13(10):983-992

6. Nilsson S, Franzen L, Parker C, Tyrrell C, Blom R, Tennvall J, Lennernas B, Petersson U, Johannessen DC, Sokal M, Pigott K, Yachnin J, Garkavij M, Strang P, Harmenberg J, Bolstad B, Bruland OS (2007) Bone-targeted radium-223 in symptomatic, hormone-refractory prostate cancer: a randomised, multicentre, placebo-controlled phase II study. Lancet Oncol 8(7):587-594 
7. Kuczyk MA, Parker C, Heinrich D, O'Sullivan JM, Fossa SD, Chodacki A, Demkow T, Logue JP, Seke M, Widmark A, Kramer MW, Johannessen DC, Nilsson S, Hoskin P, Solberg A, James ND, Syndikus I, Cross A, O'Bryan-Tear G, Garcia-Vargas JE, Sartor AO (2012) Overall survival benefit and safety profile of radium-223 chloride, a first-in-class alpha-pharmaceutical: Results from a phase III randomized trial (ALSYMPCA) in patients with castrationresistant prostate cancer (CRPC) with bone metastases. Onkologie $35: 182$

8. Scher HI, Fizazi K, Saad F, Taplin ME, Sternberg CN, Miller K, de Wit R, Mulders P, Chi KN, Shore ND, Armstrong AJ, Flaig TW, Flechon A, Mainwaring P, Fleming M, Hainsworth JD, Hirmand M, Selby B, Seely L, de Bono JS (2012) Increased survival with enzalutamide in prostate cancer after chemotherapy. N Engl J Med 367(13):1187-1197

9. Higano CS, Schellhammer PF, Small EJ, Burch PA, Nemunaitis J, Yuh L, Provost N, Frohlich MW (2009) Integrated data from 2 randomized, double-blind, placebo-controlled, phase 3 trials of active cellular immunotherapy with sipuleucel-T in advanced prostate cancer. Cancer 115(16):3670-3679

10. Kantoff PW, Higano CS, Shore ND, Berger ER, Small EJ, Penson DF, Redfern CH, Ferrari AC, Dreicer R, Sims RB, Xu Y, Frohlich MW, Schellhammer PF (2010) Sipuleucel-T immunotherapy for castration-resistant prostate cancer. N Engl J Med 363(5):411-422

11. Kantoff PW, Schuetz TJ, Blumenstein BA, Glode LM, Bilhartz DL, Wyand M, Manson K, Panicali DL, Laus R, Schlom J, Dahut WL, Arlen PM, Gulley JL, Godfrey WR (2010) Overall survival analysis of a phase II randomized controlled trial of a Poxviral-based PSAtargeted immunotherapy in metastatic castration-resistant prostate cancer. J Clin Oncol 28(7):1099-1105

12. Gulley JL, Arlen PM, Madan RA, Tsang KY, Pazdur MP, Skarupa L, Jones JL, Poole DJ, Higgins JP, Hodge JW, Cereda V, Vergati M, Steinberg SM, Halabi S, Jones E, Chen C, Parnes H, Wright JJ, Dahut WL, Schlom J (2010) Immunologic and prognostic factors associated with overall survival employing a poxviral-based PSA vaccine in metastatic castrate-resistant prostate cancer. Cancer Immunol Immunother 59(5):663-674

13. Robert C, Thomas L, Bondarenko I, O`Day S, M DJ, Garbe C, Lebbe C, Baurain JF, Testori A, Grob JJ, Davidson N, Richards J, Maio M, Hauschild A, Miller WH Jr, Gascon P, Lotem M, Harmankaya K, Ibrahim R, Francis S, Chen TT, Humphrey R, Hoos A, Wolchok JD (2011) Ipilimumab plus dacarbazine for previously untreated metastatic melanoma. N Engl J Med 364(26):2517-2526

14. Chapman PB, Hauschild A, Robert C, Haanen JB, Ascierto P, Larkin J, Dummer R, Garbe C, Testori A, Maio M, Hogg D, Lorigan P, Lebbe C, Jouary T, Schadendorf D, Ribas A, O'Day SJ, Sosman JA, Kirkwood JM, Eggermont AM, Dreno B, Nolop K, Li J, Nelson B, Hou J, Lee RJ, Flaherty KT, McArthur GA (2011) Improved survival with vemurafenib in melanoma with BRAF V600E mutation. N Engl J Med 364(26):2507-2516

15. Furie R, Petri M, Zamani O, Cervera R, Wallace DJ, Tegzova D, Sanchez-Guerrero J, Schwarting A, Merrill JT, Chatham WW, Stohl W, Ginzler EM, Hough DR, Zhong ZJ, Freimuth W, van Vollenhoven RF (2011) A phase III, randomized, placebo-controlled study of belimumab, a monoclonal antibody that inhibits B lymphocyte stimulator, in patients with systemic lupus erythematosus. Arthritis Rheum 63(12):3918-3930

16. Navarra SV, Guzman RM, Gallacher AE, Hall S, Levy RA, Jimenez RE, Li EK, Thomas M, Kim HY, Leon MG, Tanasescu C, Nasonov E, Lan JL, Pineda L, Zhong ZJ, Freimuth W, Petri MA (2011) Efficacy and safety of belimumab in patients with active systemic lupus erythematosus: a randomised, placebo-controlled, phase 3 trial. Lancet 377(9767):721-731
17. Furie R, Petri MA, Wallace DJ, Ginzler EM, Merrill JT, Stohl W, Chatham WW, Strand V, Weinstein A, Chevrier MR, Zhong ZJ, Freimuth WW (2009) Novel evidence-based systemic lupus erythematosus responder index. Arthritis Rheum 61(9):1143-1151

18. Ferlay J, Autier P, Boniol M, Heanue M, Colombet M, Boyle P (2007) Estimates of the cancer incidence and mortality in Europe in 2006. Ann Oncol 18(3):581-592

19. Eisenberger MA, Blumenstein BA, Crawford ED, Miller G, McLeod DG, Loehrer PJ, Wilding G, Sears K, Culkin DJ, Thompson IM Jr, Bueschen AJ, Lowe BA (1998) Bilateral orchiectomy with or without flutamide for metastatic prostate cancer. N Engl J Med 339(15): 1036-1042

20. Crawford ED, Eisenberger MA, McLeod DG, Spaulding JT, Benson R, Dorr FA, Blumenstein BA, Davis MA, Goodman PJ (1989) A controlled trial of leuprolide with and without flutamide in prostatic carcinoma. N Engl J Med 321(7):419-424

21. Jerant AF, Johnson JT, Sheridan CD, Caffrey TJ (2000) Early detection and treatment of skin cancer. Am Fam Physician 62(2):357-68, 375-6, 381-2

22. Avril MF, Aamdal S, Grob JJ, Hauschild A, Mohr P, Bonerandi JJ, Weichenthal M, Neuber K, Bieber T, Gilde K, Guillem V, Porta J, Fra J, Bonneterre P, Saiag D, Kamanabrou H, Pehamberger J, Sufliarsky JL, Gonzalez Larriba A, Scherrer Y (2004) Menu fotemustine compared with dacarbazine in patients with disseminated malignant melanoma: a phase III study. J Clin Oncol 22(6):1118-1125

23. Bedikian AY, Millward M, Pehamberger H, Conry R, Gore M, Trefzer U, Pavlick AC, DeConti R, Hersh EM, Hersey P, Kirkwood JM, Haluska FG (2006) Bcl-2 antisense (oblimersen sodium) plus dacarbazine in patients with advanced melanoma: the Oblimersen Melanoma Study Group. J Clin Oncol 24(29):4738-4745

24. Chapman PB, Einhorn LH, Meyers ML, Saxman S, Destro AN, Panageas KS, Begg CB, Agarwala SS, Schuchter LM, Ernstoff MS, Houghton AN, Kirkwood JM (1999) Phase III multicenter randomized trial of the Dartmouth regimen versus dacarbazine in patients with metastatic melanoma. J Clin Oncol 17(9):2745-2751

25. Middleton MR, Grob JJ, Aaronson N, Fierlbeck G, Tilgen W, Seiter S, Gore M, Aamdal S, Cebon J, Coates A, Dreno B, Henz M, Schadendorf D, Kapp A, Weiss J, Fraass U, Statkevich P, Muller M, Thatcher N (2000) Randomized phase III study of temozolomide versus dacarbazine in the treatment of patients with advanced metastatic malignant melanoma. J Clin Oncol 18(1):158-166

26. Agarwala SS (2009) Current systemic therapy for metastatic melanoma. Expert Rev Anticancer Ther 9(5):587-595

27. Julia F, Thomas L, Dumontet C, Dalle S (2010) Targeted therapies in metastatic melanoma: toward a clinical breakthrough? Anticancer Agents Med Chem 10(9):661-665

28. Lau CS, Mak A (2009) The socioeconomic burden of SLE. Nat Rev Rheumatol 5(7):400-404

29. Cheema GS, Roschke V, Hilbert DM, Stohl W (2001) Elevated serum B lymphocyte stimulator levels in patients with systemic immune-based rheumatic diseases. Arthritis Rheum 44(6):13131319

30. Petri M, Stohl W, Chatham W, McCune WJ, Chevrier M, Ryel J, Recta V, Zhong J, Freimuth W (2008) Association of plasma B lymphocyte stimulator levels and disease activity in systemic lupus erythematosus. Arthritis Rheum 58(8):2453-2459

31. Zhang J, Roschke V, Baker KP, Wang Z, Alarcon GS, Fessler BJ, Bastian H, Kimberly RP, Zhou T (2001) Cutting edge: a role for B lymphocyte stimulator in systemic lupus erythematosus. J Immunol 166(1):6-10

32. Browning JL (2006) B cells move to centre stage: novel opportunities for autoimmune disease treatment. Nat Rev Drug Discov 5(7):564-576 